\title{
De invasores a ciudadanos: la refundación de las identidades en los Pedregales de Coyoacán
}

Patricia Safa

CIESAS-OCCIDENTE

A partir del caso de las colonias populares de los Pedregales de Coyoacán en la ciudad de México, se analiza la identidad de un grupo que recurre a la "memoria" como una construcción social y cultural intencionada.

INTRODUCCIÓN

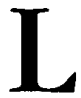

a historia oral y las memorias personales o colectivas han interesado a antropólogos e historiadores como medios privilegiados para acercarse al estudio de las sociedades y grupos que no han tenido voz propia en la historia, ${ }^{1}$ pero también por su valor etnográfico pues nos permiten acercarnos al estudio de "cómo la gente interpreta sus vidas", "se ven a ellos mismos"3 y se

${ }^{1}$ Portelli, "Pecularities", 1981.

${ }^{2}$ Rosaldo, "Reimaginando", 1992, p. 97. "imaginan lo que son o desean ser". Es decir, representan un recurso privilegiado para estudiar el proceso donde se construye el sentido, se organiza el mundo y se establecen las normas y valores que orientan el comportamiento individual y colectivo.

En el contexto de transformación urbana y de complejidad creciente, para el estudio de cómo las personas construyen el sentido de pertenencia a un lugar no interesa tanto medir el grado de transformación de estos

\footnotetext{
${ }^{3}$ Myerhoff, "Life", 1982, p. 103.

"Basso, "Stalking", 1983, p. 19.
} 
espacios, sino estudiar lo que significan para las personas en esta historia interminable de continuos cambios y reacomodos. En el análisis de la identidad vecinal como una etnografía de la experiencia, como afirma Anthony Cohen, ${ }^{5}$ "interesa estudiar cómo las personas dan sentido a esta diferencia y a partir de ella se organizan los procesos sociales en un lugar". Para definir un barrio y un vecindario tenemos que considerar sus manifestaciones objetivas, las delimitaciones geopolíticas, la organización económica y política interna y las relaciones sociales de vecindad, pero también analizar tanto la experiencia de pertenecer a un lugar como la organización vecinal para su preservación o cambio. La gente puede identificar un barrio porque puede diferenciarlo de otros por sus tradiciones o por su historia, pero sobre todo por ser un referente constructor de identidades.

Las identidades sociales se transforman con el tiempo, y por lo mismo, para crear y recrear la identidad de un grupo se recurre a la memoria que es una construcción social y cultural intencionada, "invenciones" ${ }^{6}$ que seleccionan momentos del pasado para explicar situaciones en el presente $\mathrm{e}$

s Cohen, Identity, 1986, p. 1.

${ }^{6}$ En el sentido que le da Eric.Hobsbawn, como "conjunto de prácticas, normalmente reguladas por reglas tácitamente aceptadas y de naturaleza ritual o simbólica que busca inculcar ciertas normas de comportamiento con referencia al pasado", Hobsbawn, Invention, 1983, p. 1. imaginar el futuro. Dadas las características de las relaciones de poder en la toma de decisiones sobre la direccionalidad de estos cambios, cuando se recuerda también se valora el pasado, lo que el grupo es o lo que desea ser. Este ejercicio de búsqueda de significados pertenece a una clase especial de experiencia, la reflexiva, que supone la ruptura con la vida diaria y que busca no reconstruir hechos reales sino dar significado a la vida y al mundo. En este trabajo me interesa profundizar en esta temática a través del análisis de cómo los habitantes de las colonias populares que se formaron por invasión en la década de los setenta en la zona de los Pedregales de Coyoacán, interpretan hoy en día esa experiencia y se organizan para satisfacer las carencias urbanas que aún enfrentan, pero sobre todo porque les interesa alcanzar una calidad de vida que históricamente les ha sido negada en parte por vivir en una de las ciudades más grandes e inequitativas del mundo: la ciudad de México. Para cumplir con este objetivo presentaré primero la historia de formación de las colonias para posteriormente analizar tres proyectos comunitarios: el Parque Huayamilpas, la Casa de la Cultura de Santo Domingo y las Comunidades Eclesiales de Base, como espacios que permiten a los vecinos interpretar la identidad de invasores que los unió en el pasado pero que, junto con la colonia, se ha transformado con el tiempo, dando lugar a una serie de alternativas donde hoy en día se construye el sentido de lo vecinal. 
LA INVASIÓN, UNA ALTERNATIVA

DE LOS SECTORES POPULARES PARA ACCEDER A LA VIVIENDA

El papel central de la ciudad de México en la economía y la política del país le ha permitido beneficiarse del presupuesto federal y de las obras de modernización urbana. Sin embargo, la distribución de estos beneficios no ha sido equitativa. Como algunos autores consideran, no es el tamaño de la ciudad "lo que determina esta diferenciación social y espacial sino las condiciones políticas, económicas y sociales en que se desennuelven"? Servicios urbanos y vivienda han sido las principales demandas de los sectores populares y la base para la formación de sus organizaciones vecinales.

La ciudad de México es un espacio de importantes movimientos sociales y vecinales de distinta índole que han buscado solucionar el problema de vivienda de los sectores más desfavorecidos y mejorar la calidad de vida de los habitantes de las grandes urbes. En los años veinte, por ejemplo, los moradores de vecindades y casas de alquiler ubicadas en el centro de la ciudad de México se organizaron, frente al aumento indiscriminado de la renta, para protestar por el deterioro de sus viviendas y edificios y para evitar el desalojo arbitrario. ${ }^{8}$ Hasta la fecha, el creciente deterioro de los antiguos barrios y colonias como La Lagunilla, Tepito, Peralvillo, Los Ángeles, todas ellas colonias populares en

\footnotetext{
${ }^{7}$ Ramírez, Política, 1987, pp. 389-392.

"Saucedo, "Informe", 1926.
}

el centro de la ciudad, 9 entre otros, ha permitido la organización vecinal para mejorar su entorno urbano y oponerse a planes de desarrollo urbano que ponen en peligro no sólo sus viviendas sino la transformación de un estilo de vida que les es característico. ${ }^{10}$ Este tipo de /grupos ha dado lugar a asociaciones vecinales de muy distinta índole: habitantes de un edificio, unión de residentes, asociaciones de vecinos, cooperativas de construcción, entre otras. ${ }^{11}$

La migración masiva campo-ciudad que comenzó a experimentarse en nuestro país gracias al desarrollo industrial, agudizó los problemas no resueltos de vivienda para los sectores más desfavorecidos. Lo anterior alentó la formación de una serie de colonias en la periferia de la ciudad que fue caldo de cultivo para la formación de movimientos y organizaciones populares muy diversas. En un primer momento (1968-1975) las movilizaciones populares eran aisladas, locales y con escasa coordinación en el ámbito regional o nacional. La mayoría se convirtió en clientes de organizaciones oficiales a quienes, a cambio de la satisfacción de ciertas necesidades básicas, se les pedía apoyo para legitimar las organizaciones populares del PRI. Una vez resuelto el problema estas organizaciones se desvanecían, ya que su movilización era de carácter muy coyuntural. Algunos grupos independientes lograron permanecer y

\footnotetext{
9 Coulomb, "Organizaciones", 1986; Hernández, "Tepito", 1986.

${ }^{10}$ Mercado, "Resistencia", 1986, p. 265.

${ }^{11}$ Pérez, "Lucha", 1986, p. 30.
} 
son los que, a principios de los años ochenta, forman la Conamup (Confederación Nacional del Movimiento Urbano Popular). Esta organización se caracterizó por la heterogeneidad de grupos y tendencias ideológicas, aunque los partidos políticos, sobre todo los de izquierda, han tenido una influencia indirecta en su conducción. ${ }^{12}$ El beneficio de la pluralidad, sin embargo, llevó a continuas facciones, lo que limitó las posibilidades de trascender las demandas concretas para formar un plan definido de acciones que buscara atender los problemas de la ciudad en su conjunto con programas explícitos de gestión urbana. ${ }^{13}$ Como señala Peter Ward, estos movimientos no se han constituido en amenazas reales para el Estado o para la lógica de uso del suelo capitalista, protagonistas de hecho en el crecimiento y desarrollo de la ciudad. ${ }^{1 / 4}$ No se puede desconocer, sin embargo, la importancia del MUP en la satisfacción de la demanda popular de vivienda y servicios, y en la lucha por la democratización de la ciudad. Como señala Juan Manuel Ramírez Saíz, ${ }^{15}$ entre los logros del MUP para los habitantes de las colonias populares está la resolución parcial de sus demandas, como son la obtención de terrenos, la regularización de tierra y la dotación de vivienda, la instalación y dotación de servicios, el freno a la represión, al desalojo y a la reubicación de grupos.

${ }^{12}$ Ramírez, "Movimiento", 1986.

${ }^{13}$ Ramírez, Política, 1987 , pp. 33-34, y Camino, 1994, p. 18.

${ }^{14}$ Ward, México, p. 131.

${ }^{15}$ Ramírez, Política, 1987.

\section{LA HISTORIA DE UN ACONTECIMIENTO}

El 3 de septiembre de 1971, a dos días del informe presidencial de Luis Echeverría, miles de familias invadieron terrenos comunales de Los Reyes, Coyoacán. Ya en 1968 las amenazas de invasión eran constantes pero se evitaron mediante la organización de los comuneros y la desaprobación del Estado a estas tentativas. Ese día de septiembre, los comuneros no pudieron controlar ni rechazar una invasión tan numerosa y organizada que daría lugar a la colonia Santo Domingo de los Reyes en Coyoacán. El Estado, a dos días de las declaraciones sobre los "derechos de los pobladores marginales", tampoco pudo intervenir con una represión abierta. Políticamente la situación estaba a favor de los invasores, que querían dejar de ser "arrendatarios de un cuartito" y buscaban la seguridad de poseer un lugar propio.

Los pedregales, desde hacía varias décadas, habían sido paulatinamente ocupados por sectores populares. La colonia Ajusco, por ejemplo, la más antigua de los Pedregales, se fue poblando desde los años cuarenta, cuando algunos vecinos de La Candelaria comenzaron a construir sus casas en la zona. En 1958 se llevó a cabo la primera invasión, y ya para 1960 se habían establecido en el lugar unas 2000 personas, y en 1962 como $3500 .{ }^{16} \mathrm{La}$ gente de estas colonias, sin embargo, distingue entre las dos invasiones. Considera que la invasión de la colonia Ajusco fue gradual y ordenada; la de

\footnotetext{
${ }^{16}$ Ramón, "Políticas", 1987.
} 


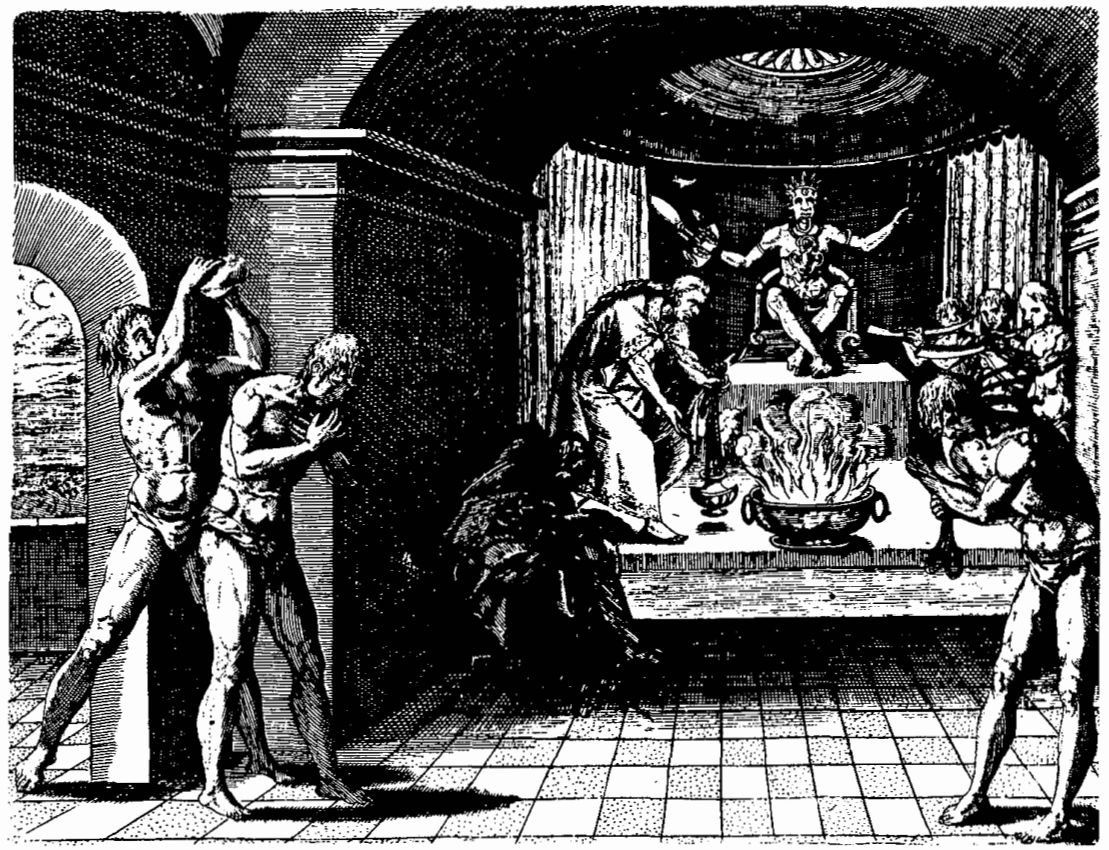

Santo Domingo, en cambio, la califican de violenta y desorganizada. ${ }^{17}$

Desde 1956, los colonos de Ajusco comenzaron a organizarse para elaborar el trazado urbano. Lo anterior permitió la formación de un conjunto de redes de ayuda mutua para la autoconstrucción de la vivienda y de la colonia, ${ }^{18}$ y la formación de un movimiento que si bien no estaba exento de conflictos internos logró convocar a la mayoría de sus habitantes. ${ }^{19}$ Los

${ }^{17}$ Safa, ePor qué?, 1992, p. 52.

${ }^{18}$ Alonso, Lucba, 1980, pp. 288-291.

19 Ibid., p. 375. pobladores de Santo Domingo también se organizaron para abrir las calles, aplanar el terreno, construir su escuela y demandar el reconocimiento de sus derechos y la dotación de servicios; sin embargo, en este segundo caso, en la medida en que la invasión fue rápida y numerosa, fue más difícil la unificación y la organización interna del movimiento. La invasión de Santo Domingo también dio lugar a una serie de enfrentamientos con los habitantes de los antiguos pueblos. Pero sobre todo, obligaron al Estado a definir una política urbana frente a los asentamientos irregulares. La 
gente se sentía amenazada por INDECO pues tenían miedo de que les quitara sus terrenos. Ante el fracaso, el asunto pasó a manos de la Procuraduría de Colonias Populares y después, en 1974, a Fideurbe (Fideicomiso de Interés Social para el Desarrollo Urbano de la ciudad de México). En un primer momento, en la colonia Ajusco, el movimiento de colonos reclamaba su derecho sobre la tierra adquirido en los años en que ocuparon los terrenos para evitar el pago de sus predios. Sin embargo, en 1976 tuvieron que negociar con el Estado el pago del terreno para convertirse en propietarios $\mathrm{y}$, posteriormente, para beneficiarse con la introducción de los servicios urbanos. ${ }^{20}$ En Santo Domingo, en cambio, el proceso de regularización fue diferente. En un primer momento, los líderes fueron los que establecieron el diálogo con el Estado. La alianza fue importante en esa etapa, pero cuando llegó la regularización, fueron rechazados. El diálogo pasó a ser entre individuos regularizados y el Estado. A pesar de estas diferencias, el proceso de incorporación a la "legalidad" estimuló la organización interna en las colonias, lo que generó la formación de movimientos urbanos populares. La población se organizaba, además, para distribuir los terrenos entre las familias. Cuando comenzaron a trazar las calles en Santo Domingo, por ejemplo, se tuvieron que reacomodar algunas familias y solicitar al gobierno nuevos terrenos para la formación de otras colonias como Huayamilpas en Coyoacán y Santo Domingo Iztapa-

\footnotetext{
${ }^{20}$ Ibid., p. 417.
}

lapa. También se formaron comités para el trazado de las calles o para negociar con FIDEURBE y con las autoridades delegacionales. Las mujeres, en este proceso, tuvieron una participación muy activa. La población trabajó para construir la colonia y colaboraron con las autoridades para asfaltar las calles e introducir agua potable y drenaje. En Santo Domingo, la población también se organizó para construir una escuela, una lechería y una tienda de abastos. Por lo mismo, consideran que gracias a ellos existe su colonia pues pagaron el costo -con trabajo y con dinero- de su consolidación. Los conflictos fueron parte de este proceso de autoconstrucción por los enfrentamientos entre los distin. tos grupos, pues muchas veces se afectaban intereses particulares: "de repente estaba una casa y el dueño salía con pistola en mano y nos decía 'pues por aquí no pasan' y ni modo... por eso las calles tienen tantas curvitas...". ${ }^{21}$

Posteriormente, este tipo de grupos pasó a formar parte de las organizaciones populares regionales y nacionales que buscaron atender las demandas de vivienda y servicios urbanos de los sectores populares de la Zona Metropolitana de la ciudad de México (ZMCM). En el caso de Santo Domingo, por ejemplo, hasta la fecha existe comunicación con organizaciones vecinales de otras colonias populares como el Ajusco, Padierna, 2 de Octubre y Santa Úrsula (declaración de Fernando Encizo).

${ }^{21}$ Fernando Encizo, director de la Casa de la Cultura de Santo Domingo. 


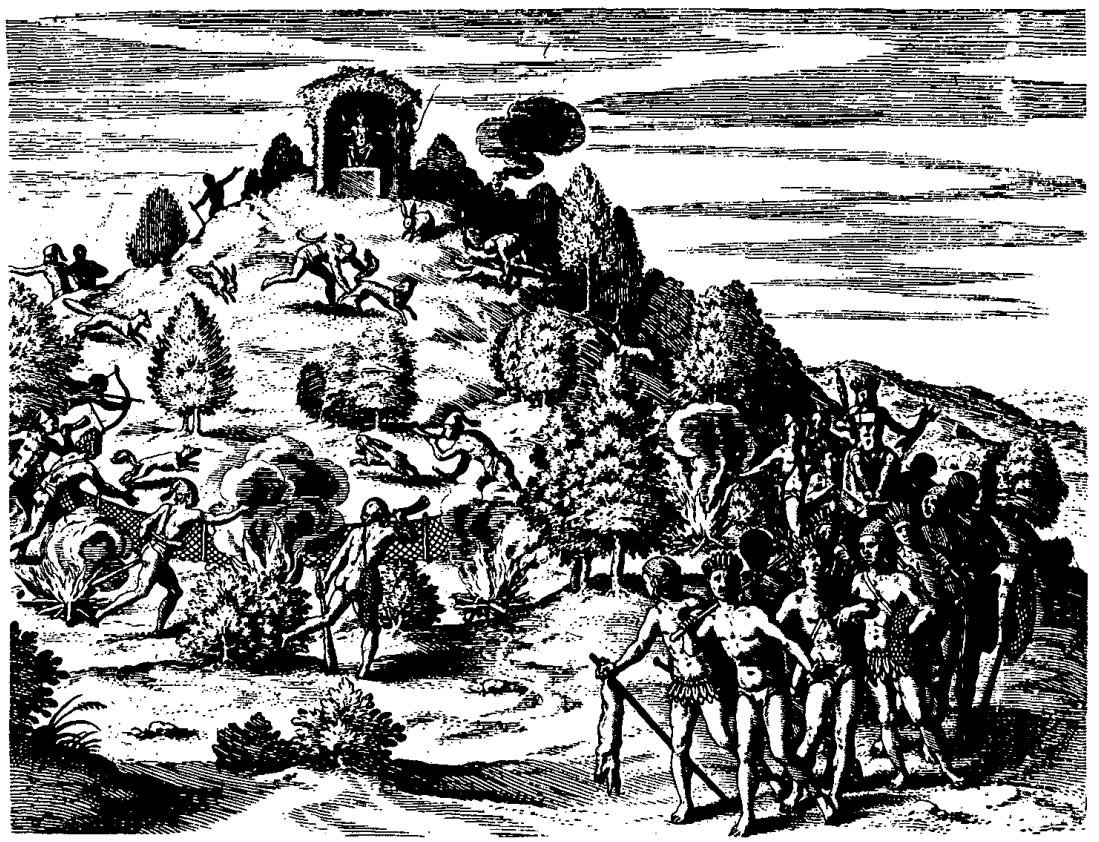

MEMORIA Y OLVIDO: LA REFUNDACIÓN DE IDENTIDADES

En la actualidad, las dos colonias cuentan con todos los servicios y, gracias a los ejes viales, al sistema de transporte urbano y a la introducción del metro en Santo Domingo, se localizan en una de las áreas mejor equipadas de la ciudad. La población de estas colonias también ha cambiado. ${ }^{22}$ Las familias que durante la invasión eran

${ }^{22}$ En un estudio que realizó Graciela Ramón Pérez en 1992, detectó que sólo $55 \%$ de las 176 familias que entrevistó habían llegado a jóvenes, actualmente han envejecido. En esa época la mayoría no habían estudiado o concluido la escuela primaria, ahora sus hijos tienen una profesión o por lo menos han estudiado hasta la secundaria. Todos estos cambios han transformado la autopercepción de sus habitantes, pues de sentirse "invasores" ahora se consideran pobladores de una colonia popular que se ubica en el "centro" de la ciudad. La identidad de invasor que sirvió para formar grupos de solidaridad y de lu-

la colonia Ajusco durante la invasión, Ramón, "Políticas", 1992, p. 32. 
cha para ellos, también significa pobreza y marginalidad urbana y social, por eso en la actualidad la rechazan:

Nos decían invasores, no lo éramos. Llegamos a un espacio feo y que nadie quería. Nosotros pagamos por la tierra, por los servicios y todo. Nuestro trabajo es lo que ha convertido a este espacio en lo que es. Invasor es quien llega a ocupar la casa de otro. Esto estaba vacío, nadie lo ocupaba, nadie nos Io regaló, nos costó a nosotros. Antes te matabas por un pedazo de tierra, ahora es una colonia como cualquiera. Ya no hay pandillas, los jóvenes también han crecido y ya tienen familia. Ahora aquí vive gente con estudios y ya no existen terrenos vacíos. Muchos, los que no pudieron pagar la regularización y los servicios, se han ido (Irene Hernández, habitante de los Pedregales).

Desde su punto de vista, lo vecinal "no es como antes". Ellos distinguen diferentes etapas: de la lucha por la tierra se pasa a la organización para obtener servicios $\mathbf{y}$, posteriormente, lo vecinal se reduce a gestiones sobre problemas concretos. Consideran que antes los unía un proyecto de colonia y de vivienda; ahora, en cambio, lo vecinal gira en torno a problemas como: "no pasa el camión de la basura", "se fue la luz", "este vecino causa problemas"... En el periodo de formación de las colonias, el conjunto de los pobladores se involucró en el movimiento. Concluido el proceso de regularización, y la introducción de los servicios urbanos, este movimiento se fracciona. En la actualidad, no todos los vecinos participan en las organizaciones populares. Algunas de estas organizaciones han desaparecido y las que no, además, para trascender el nivel particular de sus demandas, han buscado participar en organizaciones más amplias que se proponen no sólo satisfacer las necesidades de los sectores populares en materia de vivienda y servicios sino involucrarse en otro tipo de luchas de la sociedad civil como son el respeto y el derecho al voto, el control de la ciudadanía sobre el gobierno, alternativas a la política económica, la seguridad pública, la paz en el país, el cuidado del medio ambiente o la intervención en el diseño de las políticas públicas. ${ }^{23}$

\section{DE COLONOS A VECINOS}

En 1977 el regente Hank González comenzó a utilizar la estructura de las juntas de vecinos. El objetivo era que los ciudadanos se sintieran satisfechos con las oportunidades de representación local. Fue en este periodo cuando se creó la figura de jefes de manzana y de presidentes de asociación de residentes. Esta estructura de participación vecinal, sin embargo, hasta la fecha no ha logrado tener mayores alcances, ya que se ha convertido en instrumento de control vecinal de las autoridades locales. ${ }^{24}$ Anaya Lazúrtegui señala, por ejemplo, que si bien la función de un presidente vecinal es atender las demandas sentidas de la población y crear nexos de relación entre la autoridad y los habitantes de la ciudad, de hecho ha funcionado "como el aparato avalador de las decisiones de la autoridad y como cuerpo

${ }^{23}$ Ramírez, "Movimiento", 1996, p. 295.

${ }^{24}$ Ward, México, 1991, p. 126. 
de acompañantes distinguidos en las giras de delegados y funcionarios públicos". ${ }^{25}$ A lo largo de todos estos años, la participación de la población en la elección de los representantes vecinales ha sido casi nula. Las juntas locales de vecinos son cuestionadas ya que sus funciones son sólo de consulta y opinión, persistiendo la autoridad decisoria y ejecutiva del delegado correspondiente que no necesariamente se interesa en promover la participación de los vecinos, ni en escuchar las demandas canalizadas por estos conductos. ${ }^{26}$

Las autoridades locales han controlado en buena parte a los presidentes vecinales, y en su mayoría pertenecen al PRI. Por ejemplo, la señora Dolores Navarro, ex presidenta de la Junta de Vecinos de Huayamilpas, trabajó primero como presidenta de su colonia en los Comités del DIF (Instituto de Desarrollo Integral de la Familia). Fue entonces cuando aprendió a coordinar grupos populares. El ex presidente de la Junta de Vecinos en la colonia Ajusco también pertenece al PRI desde hace 16 años. Llegó a vivir a la colonia cuando era niño, hace 34 años, y su padre fue secretario de la Asociación de Colonos en los setenta. Cuando era más joven fue el secretario de la juventud popular revolucionaria de la CNOP. Comentaba que en las elecciones de 1988 el partido no ganó en este distrito y que por lo mismo les interesó comenzar a participar activamente en las organizaciones vecinales para recuperar el voto

\footnotetext{
${ }^{25}$ Anayá, "Ciudadanización", 1995, p. 4.

${ }^{26}$ Rodríguez, "Gobierno", 1987, p. 379.
}

de la población. Cuando fue presidente de la Junta de Vecinos se encargó de atender, por ejemplo, los problemas de regularización de las familias que todavía no poseen escrituras de sus terrenos ${ }^{27}$ y de resolver los problemas cotidianos de la comunidad. Para realizar su tarea, fue importante colaborar con el programa Solidaridad, ${ }^{28}$ sobre todo porque lo que a él le interesaba era volver a ganar el voto de la gente. También promovió la relación con otros presidentes vecinales de las colonias populares contiguas para que ganaran los que pertenecen al PRI. Nos comentaba, por ejemplo, cómo apoyó la candidatura de Carlos Sánchez para presidente de La Candelaria para "evitar problemas entre nosotros". En las elecciones de 1996 sólo perdieron en la colonia Santo Domingo, en la Ruiz Cortines y en Huayamilpas, que fueron ganadas por grupos de oposición. Aunque formalmente este tipo de organización no es partidario, de hecho lo es y las alianzas y conflictos entre partidos se manifiestan como problemas entre grupos de vecinos. Ser presidente vecinal, además, es un puesto por el que compiten los militantes de los partidos pues se considera un escalafón en la carrera políti-

\footnotetext{
${ }^{27}$ Comentaba que en la colonia todavía existen 300 o 400 lotes irregulares y que el $20 \%$ de las casas en la colonia no cuentan con drenaje.

${ }^{2 B}$ El programa fue creado durante el sexenio de Carlos Salinas de Gortari para desarrollar programas sociales de apoyo a los sectores populares. Este tipo de programas ha servido para "asegurar" votos al partido oficial, pues a cambio del apoyo electoral, ofrece recursos para desarrollar programas de mejoras urbanas.
} 
ca. Por lo anterior, a veces la gente no reconoce a sus representantes en la medida en que consideran que lo que a ellos les interesa son las elecciones y cuidar su presencia política en la zona y no los problemas reales de la comunidad. De hecho, las autoridades locales atienden las demandas vecinales sobre todo si sus representantes pertenecen al partido en el poder. Su respuesta también depende de la capacidad que tenga el grupo para negociar con él o presionarlo. En algunos casos los ignora, no asiste a sus reuniones o manda a uno de sus subordinados a negociar con ellos. El presidente de la Junta de Vecinos de la colonia Ajusco comentaba que muchas veces no puede hacer citas para negociar con el delegado, aunque "es su derecho el que lo atienda y obtener recursos para las obras que se han propuesto en la colonia". Reconoce, sin embargo, que gracias a las autoridades ha podido gestionar la introducción de servicios como el drenaje o regularizar las escrituras.

Las autoridades locales promueven políticas encaminadas a atender las demandas de las colonias populares a través de diferentes programas de participación comunitaria. Este tipo de programas ha cambiado de nombre. El programa anterior se llamaba Parte y Parte, y en él se establecía que el vecino ponía la mano de obra y el material para las banquetas y el gobierno la asesoría de la obra. Ahora este programa se llama Solidaridad. Este programa apoya a los vecinos para que pinten sus edificios, para introducir servicios o para construir sus viviendas. Sin embargo, como considera el presidente de la Junta de Vecinos de Ajusco, la "solidaridad entre los vecinos no es novedosa, siempre lo hemos hecho; la nueva solidaridad es con los gobernantes" ya que, como la misma gente opina, "el poder no es monolítico, tiene sus fracturas y, en ciertas coyunturas, se puede negociar con ellos y obtener algunos beneficios" (Fernando Encizo). En la actualidad, con la elección de Cuauhtémoc Cárdenas como regente de la ciudad, en las distintas delegaciones los partidos políticos que cuentan con base territorial esgrimen luchas cotidianas por consolidar y legitimar su posición. En este rejuego de poder, los recursos, los servicios, los proyectos, se han convertido en arenas de conflicto y confrontación. Algunas autoridades se preocupan por "dar voz a sus habitantes" o los convierten en socios de proyectos, como es el caso del parque Huayamilpas que se analizará posteriormente.

A pesar de estos problemas, la Junta de Vecinos es una instancia que está cobrando cada vez mayor importancia en la medida en que se amplían sus facultades y poder de decisión. Sin embargo, por la falta de representatividad de la junta directiva, en los últimos años presenciamos la formación de un sinnúmero de asociaciones vecinales independientes que surgen para atender el problema del medio ambiente, para resolver un problema en particular o para apoyar la preservación y mantenimiento de espacios colectivos. La constitución de asociaciones civiles es otra alternativa para la organización vecinal a las formalmente reconocidas que se crean como respuesta ante la cada vez más deficiente 


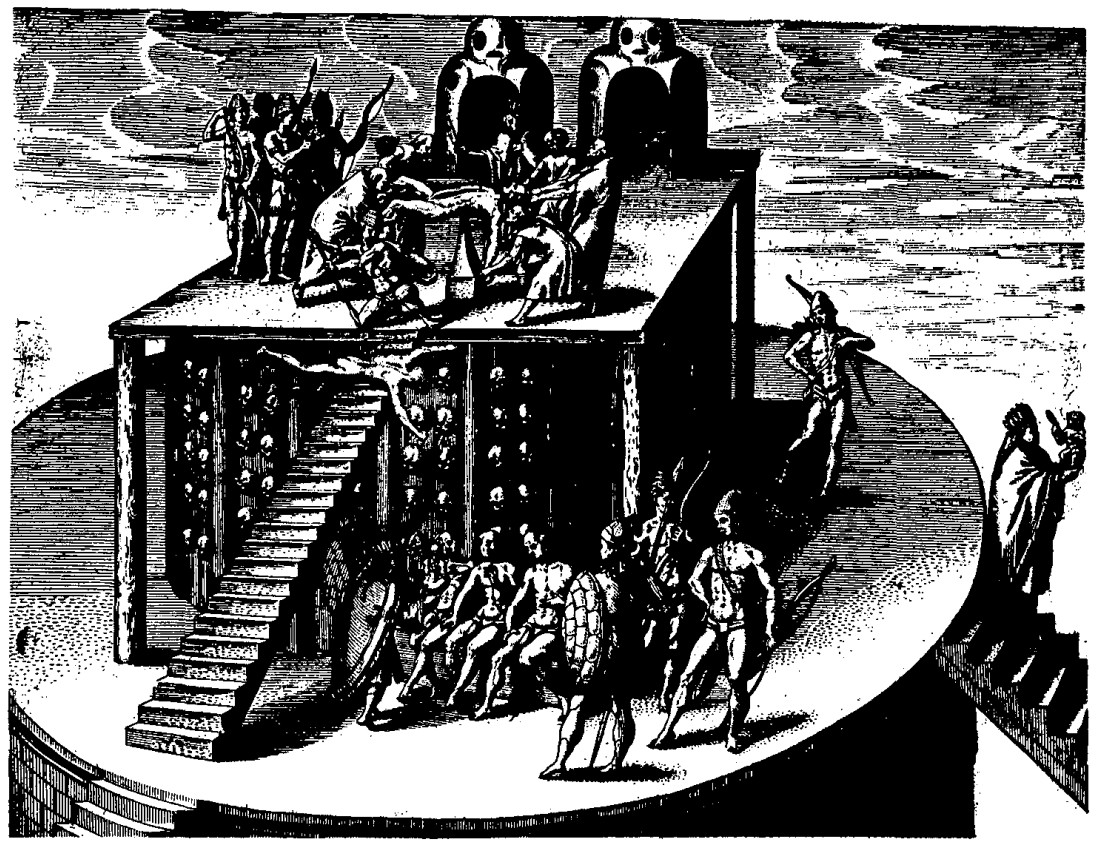

administración de la ciudad, pero también responden a las medidas tomadas por las autoridades de promover la autogestión para la solución de los problemas urbanos. En este trabajo presentaré tres proyectos comunitarios que han servido para la refundación de grupos e identidades a partir de los cuales se construye lo vecinal.

el parque Huayamilpas: UN PROYECTO COMUNITARIO

En la actualidad, los grupos vecinales no sólo solicitan apoyo para la vivien- da, servicios públicos o programas de asistencia social. La seguridad en la ciudad, el cuidado del medio ambiente, la instalación de parques, centros deportivos y culturales, son otras de sus demandas. Lo anterior ha permitido la formación de grupos muy diversos que continúan esta lucha para satisfacer sus múltiples demandas. Por lo mismo, la identidad vecinal, en un primer momento cohesionada alrededor de la invasión, se ha fragmentado dando lugar a múltiples caminos para construir los lazos de pertenencia al lugar.

Los vecinos de los Pedregales, de la misma manera como participaron en 
la autoconstrucción de la colonia, se han interesado en promover la construcción de un parque de reserva ecológica para proteger el paisaje natural de los Pedregales. Para alcanzar esta meta se organizaron en diferentes comités, para limpiar un terreno baldío que se usaba como basurero en los terrenos donde se reubicó a las familias que no encontraron un lugar durante la invasión de Santo Domingo. La gente comenzó a trabajar desyerbando la tierra, construyeron canchas deportivas y caminos para que la gente paseara. Decidieron entonces constituirse en una asociación civil. El proyecto era muy costoso y solicitaron a las autoridades que invirtieran dinero para habilitarlo y construir una casa de la cultura en el parque. El principal problema que actualmente enfrentan los vecinos es la invasión de algunos grupos de colonias vecinas que prefieren vivienda y no un parque en este terreno. También carecen de un presupuesto suficiente para evitar que se seque el lago, para darle el mantenimiento adecuado o para contratar personal y maestros para la Casa de la Cultura. Sin embargo, y a pesar de estos problemas, éste es el único parque que existe en los Pedregales. Por lo mismo, se ha convertido en un lugar de encuentro para los vecinos de la zona. Sin embargo, la comunidad reconoce que el parque existe gracias a la comunidad que logró contar con el apoyo de las autoridades locales. A la mayoría les gusta mucho su parque, el lago, el ambiente, el paisaje y sus canchas deportivas. Les gustaría que hubiera más árboles, bancas dónde sentarse, un equipo de seguridad y limpieza, arreglar las canchas y más juegos. Algunos desean tener un pequeño zoológico y una biblioteca. Proponen para ello que se cobre la entrada a un peso por persona. Por la falta de presupuesto, la delegación busca delegar la responsabilidad del parque a los vecinos o a los grupos interesados en mantener el proyecto. Lo anterior ha ocasionado divisiones internas en la comunidad. A Fernando Encizo, que coordina un proyecto de la Casa de la Cultura en Santo Domingo, le interesa retomar el proyecto pero independiente de las autoridades. Dolores Navarro, una de las promotoras del parque, considera que es mejor mantener la asociación con las autoridades para asegurar su consolidación. Hasta la fecha, el parque se mantiene y es atractivo para la población a pesar de la falta del presupuesto adecuado para su mantenimiento.

\section{la Casa de la Cultura de Santo DOMINGo}

El parque Huayamilpas surgió como iniciativa de la comunidad, al igual que la Casa de la Cultura que coordina Fernando Encizo en Santo Domingo. Fernando vivía en Copilco Bajo, Coyoacán, cuando ocurrió la invasión. En 1968 participó en el movimiento estudiantil de la UNAM y se incorporó a grupos que buscaban "contribuir en mejorar las condiciones del pucblo". Por lo mismo, le correspondió apoyar al grupo de invasores. "Le gustó" Santo Domingo y decidió participar como invasor para obtener un terreno en la zona. Como parte del grupo, partici- 
pó activamente en la formación de los comités y de la Unión de Colonos. A él te tocó, por ejemplo, participar en el reacomodo de la población en Huayamilpas y en Santo Domingo Iztapalapa. Cuando terminó el proceso de regularización siguió trabajando en un proyecto educativo para jóvenes, que es el antecedente de la actual Casa de la Cultura. Este centro se inauguró en 1993 en la antigua escuela que los colonos construyeron durante la invasión. El proyecto nació "para ofrecer espacios de reunión a la gente", de los que la colonia carece. El grupo quería abrir un centro cultural con foro abierto, cafetería, biblioteca, aulas e imprenta. Para conseguir dinero solicitaron el apoyo del DDF con el cual firmaron un convenio de colaboración. Manuel Camacho, en ese entonces regente de la ciudad, financió la construcción del edificio y el grupo se comprometió a aportar maestros y material de trabajo. La única condición que impuso el grupo fue que el proyecto se mantuviera independiente. Los maestros son estudiantes universitarios que prestan su servicio social y voluntarios de la misma comunidad. En el centro se ofrecen desayunos o comidas muy baratas (un peso por cada uno). Con ese dinero compran alimentos y se da una propina a los cocineros. También cuentan con una imprenta que usan para capacitar a jóvenes y para elaborar anuncios o comunicados de la organización. Tienen un taller de carpintería para enseñar a la gente a hacer sus muebles. Los maestros son carpinteros de la zona. El grupo tiene planeado rescatar una vieja cantera para con- vertirla en área de esparcimiento. En la cantera existe un barranco de 35 o 40 metros de profundidad que en el fondo tiene un pequeño lago. Habilitado, consideran, puede convertirse en un lugar de paseo muy atractivo para los habitantes de los Pedregales. A Fernando Encizo le interesa que este tipo de proyectos se mantenga bajo el control de la comunidad, aunque reconoce que requieren del apoyo económico de las autoridades para llevarse a cabo.

\section{LAS COMUNIDADES EClesiales DE BASE}

Los grupos religiosos, sobre todo los católicos, son otra alternativa para la construcción de las relaciones vecinales en las colonias populares. En la década de los setenta, por ejemplo, cuando en la colonia Ajusco se formó la Unión de Colonos, un grupo de religiosos los apoyó en su movimiento. ${ }^{29}$ Cuando los colonos negociaron con el Estado la regularización de la tierra, los religiosos continuaron su trabajo en la comunidad ofreciendo, además de los servicios religiosos que ofrecen en la parroquia de la Resurrección, una serie de programas educativos y de salud. Desde la iglesia se organizan las Comunidades Eclesiales de Base (CEB) que además de formar grupos de oración realizan acciones de ayuda mutua, como apoyo y visita a los enfermos o a los más necesitados. Víctor Verdini, párroco de la iglesia,

${ }^{29}$ Alonso, Lucha, 1980, p. 381. 
considera que la gente ha cambiado mucho desde la época de la invasión. Me explicaba que si bien fue importante apoyar a los colonos cuando la colonia comenzó a formarse, ahora "se requiere de otro tipo de pastoral más efectiva para resolver sus problemas concretos". Las CEB son para él "una vivencia de fe cristiana que sirve para la vida, para vivir mejor y para construir un compromiso entre hermanos que se reúnen para hablar de cosas concretas y pequeñas".

En las Comunidades Eclesiales de Base buscamos hacer conciencia de nosotros mismos y de lo que nos rodea. Nos interesa pensar nuestros problemas a la luz del Evangelio para conocer cómo debemos actuar. No se puede separar la fe de la política. Jesús fue un político pues habló con la verdad. Hablar con la verdad fue lo que lo llevó a la cruz. (Natalia Ruiz, participante de la CEB en la colonia Ajusco.)

En las colonias populares también se organizan grupos para celebrar las fiestas del santo patrono de un barrio de la colonia o para colaborar en las fiestas de su lugar de origen. En Santo Domingo, por ejemplo, se celebra la fiesta de Cristo Rey el 20 de noviembre y la fiesta de la Asunción el 20 de marzo. En esta colonia también existen cofradías de un santo patrono especial, como la Virgen de San Juan de los Lagos y/o la Virgen de Guadalupe, que se encargan de organizar peregrinaciones a sus respectivas basílicas. Estos grupos participan en la fiesta de otros barrios y pueblos como es el caso de la fiesta del Señor de las Misericordias del pueblo de Los Reyes.

\section{COMENTARIOS FINALES}

En este trabajo fue importante superar una visión de vecindarios como comunidades homogéneas, condición para la construcción de las identidades vecinales; y considerar que si bien lo barrial y lo vecinal son, sobre todo, experiencias constructoras de identidades, también se constituyen en una arena política de negociación y confrontación.

Las identidades sociales son ese "cúmulo de representaciones compartidas que funcionan como matriz de significados que permiten definir y valorar lo que somos frente a los otros". ${ }^{30}$ Por lo mismo, no son "cosas" que pueden transmitirse de generación en generación sino "procesos de contraste", ${ }^{31}$ "sistemas de relaciones y representaciones" 32 que permiten a las personas reconocerse y ser reconocidos por otros (proceso de identificación) pero también reconocer a los otros (proceso de diferenciación). Las identidades no son estáticas, cambian con el tiempo y son resignificadas de acuerdo con los contextos históricos. En los Pedregales, lo que unió a la población para construir la colonia, con el tiempo perdió su valoración positiva transformándose para conformar nuevas identidades que dan lugar a distintos grupos vecinales interesados en cuidar el entorno y mejorar la calidad de vida de los sectores populares.

Los MUP que surgieron para atender las demandas de los sectores po-

\footnotetext{
${ }^{30}$ De la Peña, "Identidades", 1994.

31 Portal, Identidad, 1995, p. 28.

${ }^{32}$ Melucci, "Sobre", 1982, p. 9.
} 


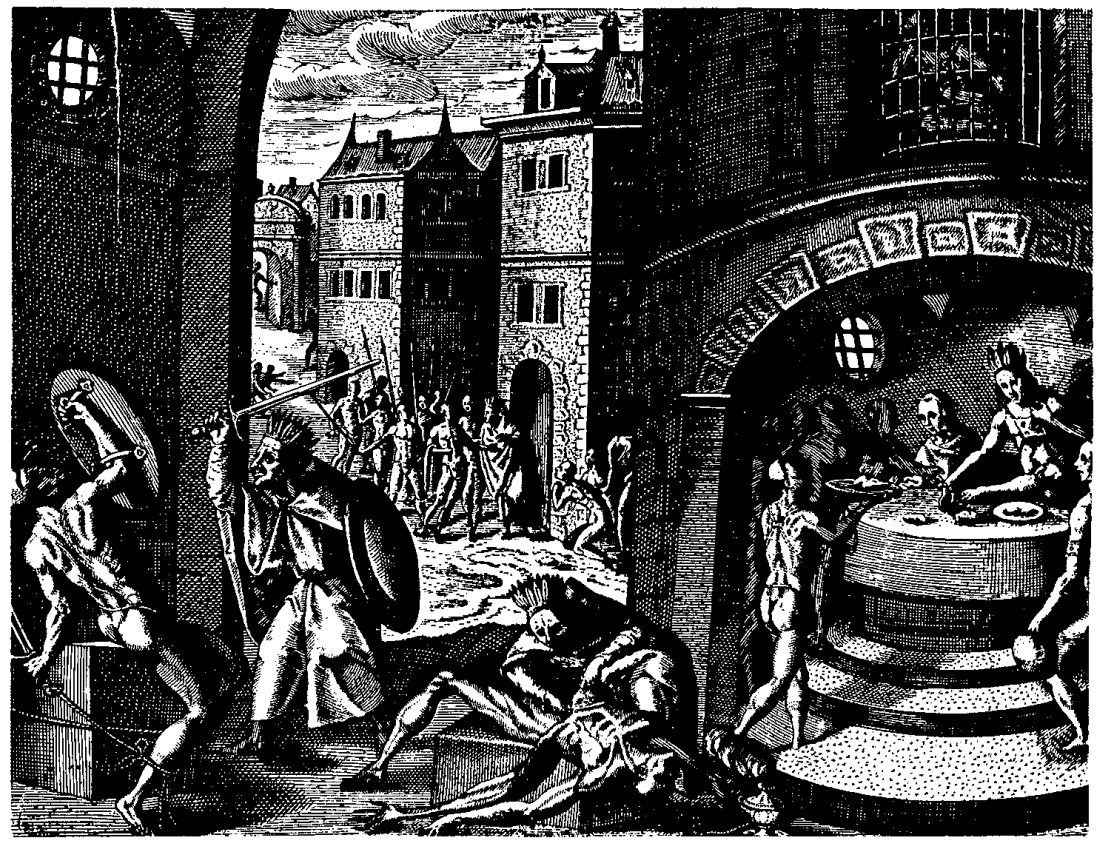

pulares, lograron articular la lucha de los más afectados por la desigual distribución de los bienes urbanos. Sin embargo, este tipo de solidaridades, las que se formaron no porque los grupos de colonos o invasores fueran homogéneos, sino porque los unía una meta compartida como la regularización de la tierra o la introducción de servicios, en la actualidad se han fraccionado dando pie a múltiples alternativas para la construcción de lo vecinal. La problemática vecinal de los sectores populares no se agota con las demandas que se generan por la de. sigual distribución de los bienes urba- nos, también les interesa que en sus colonias existan parques, centros deportivos y culturales que ofrezcan alternativas educativas y de esparcimiento a sus hijos. Les interesa mejorar la calidad de vida y se organizan para cuidar un parque o para formar asociaciones y grupos de interés particulares.

Para los sectores populares "la negociación" ha sido la principal estrategia de lucha. Por la falta de recursos han establecido alianzas con el Estado a través de sus intermediarios que buscan desarrollar una carrera política como líderes populares, o han busca- 
do la adhesión a los MUP y/o a los partidos políticos de oposición como otra de las múltiples alternativas para presionar a las autoridades para la satisfacción parcial de sus demandas. Lo anterior explica la dificultad que existe entre estos sectores para la formación y consolidación de organizaciones autónomas e independientes. Por otro lado, la lucha de los sectores populares no ha estado exenta de contradicciones y tensiones. Las redes de solidaridad han sido ocasión de pugnas entre líderes y de violencias internas, y sus demandas han servido para legitimar las políticas sociales del Estado a pesar de que ellos mismos han financiado con dinero y con trabajo el costo de la autoconstrucción de la colonia y de su vivienda. El desarrollo de proyectos independientes se explica por la falta de instancias eficaces de participación ciudadana donde la población pueda efectivamente participar en la toma de decisiones sobre la ciudad. En este contexto de diversidad las identidades articulan la organización vecinal porque permite la reflexión sobre el proyecto de vida que desean vivir. En este sentido, la búsqueda de identidades vecinales, más que una añoranza por el pasado, responde a nuevas situaciones problemáticas en el presente $\mathrm{y} / \mathrm{o}$ a proyectos de utopías futuras.

\section{BIBLIOGRAFIA}

-Alonso, Jorge (comp.), Lucha urbana y acumulación de capital, Ediciones de la Casa Chata/CIS-INAH, México, 1980. (coord.), Los movimientos sociales en el Valle de México, ciesas, México, 1986 (Col. Miguel Othón de Mendizábal).

-Anaya Lazúrtegui, Elizabeth, "Ia ciudadanización de los partidos políticos en el Distrito Federal", conferencia presentada en el XX Congreso Latinoamericano de Sociología. América Latina y el Caribe: Perspectivas de su Reconstrucción, realizado en la ciudad de México del 2 al 6 de octubre de 1995.

-Basso, Keith H., "Stalking with stories: names, places, and moral narratives among the western apache" en Edward $M$. Bruner (comp.), Text, play, and story: the construction and reconstruction of self and society, Proceedings of the American Ethnological Society/TAEL, Washington, 1983, pp. 19-55.

-Cohen, Anthony, "Belonging: the experience of culture" en Anthony Cohen (comp.), Belonging, identit and social organization en british rural cultures, Manchester University Press, Manchester,1982.

Identity and diversity in britisb culture, Manchester University Press, Manchester, 1986.

-Coulomb, René, "Organizaciones populares y planeación urbana en un barrio deteriorado de la ciudad de México" en Alonso, Movimientos, 1986, pp. 297-318.

-De la Peña, Guillermo y Renée de la Torre, "Identidades urbanas al final del milenio", Revista Ciudades: Movimiento Social y Organización Ciudadana, Red Nacional de Investigación Urbana, núm. 22, abril-junio, 1994, Puebla.

-Hernández, Alfonso, "Tepito para los tepiteños" en Alonso, Movimientos, 1986, pp. 333-346.

-Hobsbawn, Eric y Terence Ranger (comps.), The invention of tradition, Cambridge University Press, Cambridge, 1983.

-Massolo, Alejandra, "La marca del género: mujeres protagonistas de la ciudad" 
en Augusto Bolívar et al. (coords.), Gestión metropolitana y política, UAM-Azcapotzalco, México, 1994, 'pp. 419-444.

-Mellucci, Alberto, "Sobre la identidad" en L'Ivenzione del presente. Movimenti, identita, bisogni individuali, trad. de Mónica Mansour, II Mulino, Boloña, 1982, pp. 61-72.

-Mercado, Ángel, "Resistencia de pobladores en el centro de la ciudad de México" en Alonso, Movimientos, 1986.

-Myerhoff, Barbara, "Life history among the elderly: performance, visibility and remembering" en Jay Rusy (comp.), A crack in the mirror. Reflexive perspectives in antbropology, University of Pennsylvania Press, Philadelphia, 1982, pp. 99-117.

-Pérez, José Antonio, "Lucha por la vivienda en el centro de la ciudad: el edificio Gaona" en Alonso, Movimientos, 1986, pp. 319-332.

-Portal, María Ana, "Identidad urbana y religiosidad popular", tesis de doctorado, Facultad de Filosofia y Letras-UnAM, México, 1995.

-Portelli, Alessandro, "The peculiarities of oral history", History Worksbop, núm. 12, otoño, 1981, Londres, pp. 97-107.

-Ramírez Saíz, Juan Manuel, El movimiento urbano popular en México, Siglo $\mathrm{XXI} / \mathrm{Instituto}$ de Investigaciones Sociales, México, 1986.

\footnotetext{
, Política urbana y lucba popular, UAM, México, 1987.

, Los caminos de la acción colectiva, Ensayos Jalisciences/E1 Colegio de Jalisco/INAH, Guadalajara, 1994.
}

"Movimientos urbano-populares: política local y democratización en Guadalajara" en Jorge Alonso y J. M. Ramírez Saíz (comps.), La democracia de los de abajo, UdeG/CIESAS/UNAM/Consejo Electoral del Estado de Jalisco, Guadalajara, 1996, pp. 277-308.

-Ramón Pérez, Graciela, "Políticas culturales y necesidades sociales: el DIF, un programa de asistencia social", tesis, UAMIztapalapa, México, 1992.

-Rodríguez Araujo, Octavio, "Gobierno y representación política en el Distrito Federal, 1940-1984" en Atlas de la ciudad de México, El Colegio de México, México, 1987.

-Rosaldo, Renato, "Reimaginando las comunidades nacionales" en José Manuel Valenzuela Arce (comp.), Decadencia y auge de las identidades, El Colegio de la Frontera Norte, Tijuana, 1992, pp. 191-201.

-Safa Barraza, Patricia, ¿Por qué se envía a los bijos a la escuela? Socialización infantil e identidad popular, Editorial Grijalbo, México, 1992.

-Saucedo, Miguel, "Informe de gobierno que rinde el $\mathrm{C}$. presidente municipal de la villa de Coyoacán, D. F., Miguel Saucedo, sobre su gestión administrativa realizada en el periodo correspondiente del 27 de mayo al 31 de octubre de 1926", H. Ayuntamiento constitucional de la villa de Coyoacán, Coyoacán, D. F., 1926.

-Ward, Peter, México: una megaciudad. Producción y reproducción de un medio ambiente urbano, CNCA/Alianza Editorial, México, 1991 (Los Noventa). 


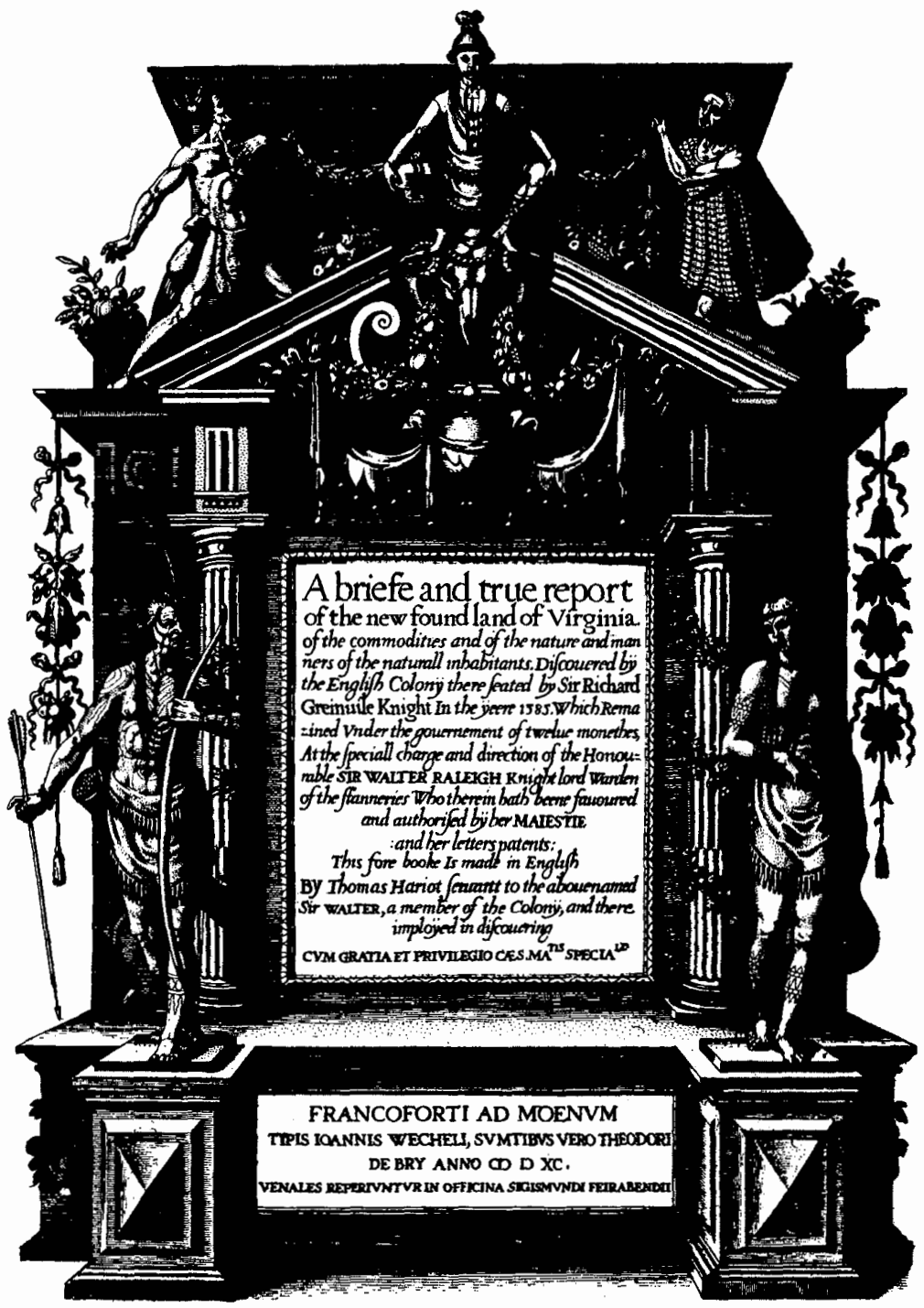

